\title{
N95 respirator or triple layer surgical mask: Radiologist perspective
}

N95 respirators and surgical masks (face masks) are examples of personal protective equipment (PPE) that are used to protect the wearer from airborne particles and from liquid contaminating the face. The respirators are designed to reduce inhalation exposure to particulate contaminants (dust, mist, and fume). The authorities which regulate the quality control for the respirators are Centers for Disease Control and Prevention (CDC), National Institute for Occupational Safety and Health (NIOSH), and Occupational Safety and Health Administration (OSHA).

\section{Surgical Mask}

A surgical mask is a loose-fitting, disposable device that creates a physical barrier between the mouth and nose of the wearer and potential contaminants in the immediate environment. Surgical masks are made in different thicknesses and with different ability to protect healthcare personnel from contact with liquids. If worn properly, a surgical mask is meant to help block large-particle droplets, splashes, sprays, or splatter that may contain germs (viruses and bacteria), keeping it from reaching to the mouth and nose. The surgical masks do not form a tight seal against the face skin and so are not recommended to protect people from airborne infectious diseases. Surgical masks have been relegated for protection against infection through fluid repellence only. The protection provided by the surgical masks against particles $(0.04-1.3 \mu \mathrm{m})$ is $8-12$ times less than N95 respirators. ${ }^{[1]}$ These masks are not intended to be used more than once.

The triple layer surgical mask is made up of a melt-blown polymer, most commonly polypropylene, placed between nonwoven fabric. ${ }^{[2]}$ The melt-blown material acts as the filter that stops microbes from entering or exiting the mask. Pleats are commonly used to allow the user to expand the mask such that it covers the area from the nose to the chin. These masks are secured to the head with ear loops, head ties, or elastic straps.

\section{N95 Respirators}

An N95 respirator is a type of disposable filtering half-facepiece respirator (DFHFR) classified as air-purifying respirator. To be a certified filtering facepiece respirator, the mask must be NIOSH approved, double strapped, and clearly labelled. The "N95" designation means that when subjected to careful testing, the respirator blocks at least $95 \%$ of very small $(0.3 \mu \mathrm{m})$ test particles. The key features of N95 masks are adjustable nose clip (for leak-proof fit and disallows fogging of eyewear), nose foam (for absorption of perspiration), and headbands. The nose clip is made of aluminum, nose foam made of polyurethane, filter made of polypropylene, and the cover made of polyester. ${ }^{[3]}$

Respirators in this family are rated as $\mathrm{N}, \mathrm{R}$, or $\mathrm{P}$ for protection against oils. $N$ (not resistant to oil) means that the respirators cannot be used in an oil droplet environment; $\mathrm{R}$ (somewhat resistant to oil) and $\mathrm{P}$ (strongly resistant to oil) mean that these respirators can be used for protection against nonoily and oily aerosols. The US National Institute for Occupational Safety and Health (NIOSH) classifies filtering facepiece respirators (FFRs) into nine categories (N95, N99, N100, P95, P99, P100, R95, R99, and R100). Numerical designations 95, 99 , and 100 show the filter's minimum filtration efficiency with $95 \%, 99 \%$, and $99.97 \%$, respectively..$^{[4,5]}$ N95 respirator may have an exhalation valve which reduces excessive dampness and warmth in the mask from exhaled breath. According to the European standards, the FFP2 respirators are equivalent to NIOSH approved N95 respirator. Table 1 below highlights where to use which mask in various sections in Radiology.

Table 1: N95 Respirator and Surgical Mask: Where to Use What?

\begin{tabular}{|c|c|c|c|c|}
\hline \multirow[t]{2}{*}{ Setting } & \multicolumn{2}{|c|}{ COVID Suspect } & \multicolumn{2}{|c|}{ COVID Positive } \\
\hline & Surgical mask* & N95 & Surgical mask & N95\# \\
\hline X-Ray & - & $\sqrt{ }\left({ }^{* *}\right)$ & - & $\sqrt{ }(* *)$ \\
\hline Ultrasound & - & $\sqrt{ }$ & - & $\sqrt{ }$ \\
\hline Mammography & - & $\sqrt{ }$ & - & $\sqrt{ }$ \\
\hline CT & - & $\sqrt{ }$ & - & $\sqrt{ }$ \\
\hline MRI & - & $\sqrt{ }$ & - & $\sqrt{ }$ \\
\hline $\begin{array}{l}\text { Intervention } \\
\text { DSA Lab }\end{array}$ & - & $\sqrt{ }$ & - & $\sqrt{ }$ \\
\hline \multicolumn{5}{|c|}{$\begin{array}{l}\text { *-Surgical mask with face shield or mask with integral visor can be used as a measure in } \\
\text { only crisis situations with adequate social distancing } \\
\left({ }^{* *}\right) \text {-Surgical mask with face shield can be an alternative if glass barrier created between } \\
\text { equipment and the patient } \\
\text { \#It is recommended that those with beards consider shaving their facial hair for an } \\
\text { appropriate N95 mask fitting. Note that N95 masks may have a metal strip, risking loss } \\
\text { of seal in the MRI room; nonferrous N95 masks may be available. N95 masks may be } \\
\text { preserved for reuse by wearing overlying surgical/isolation masks and/or face shield or by } \\
\text { central cleaning per institutional practice }{ }^{[6]}\end{array}$} \\
\hline
\end{tabular}


Comparison between N95 Respirator and Surgical Mask ${ }^{[5]}$

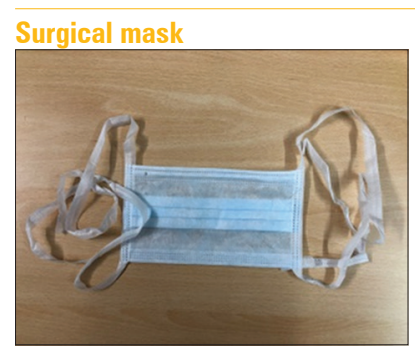

Fluid resistant - provides protection against large droplets, splashes, or sprays of bodily or other hazardous fluids

Loose-fitting

Does not require Fit Test

Does not require seal check

Does not provide a reliable level of protection from inhaling smaller airborne particles

Leakage occurs around the edge of the mask when user inhales

Disposable - discarded after each use

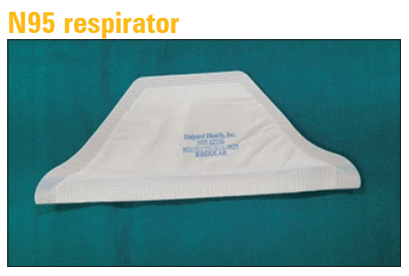

Provides protection against small particle aerosols and large droplets (only nonoil aerosols)

Tight-fitting

Requires Fit Test before use Requires seal check

Filters out at least $95 \%$ of airborne particles including large and small particles

When properly fitted and donned, minimal leakage occurs around edges of the respirator when user inhales

Ideally should be discarded after each patient encounter and after aerosol-generating procedures. It should also be discarded when it becomes damaged or deformed; no longer forms an effective seal to the face; becomes wet or visibly dirty; if it becomes contaminated with blood respiratory or nasal secretions, or other bodily fluids from patients.

During crisis situations can be reused after proper disinfection

\section{Valved vs. Nonvalved Respirators}

The most important difference between the valved and nonvalved respirator is the location of filter. In a valved respirator, the filter is located inside the valve, whereas in a nonvalved respirator, the filter is situated within the fabric itself. Valved respirators make it easier to exhale air and are thus more comfortable to wear, and leads to less moisture build-up inside the respirator. Valved respirators do not filter the wearer's exhalation and, hence, are not suitable for a COVID-19 patient. ${ }^{[7]}$ No clear cut guidelines are available if the valved respirator can be used by a healthcare worker inside the hospital; however, putting a surgical mask over the valved respirator can provide protection to others especially when there is community transmission. Valved respirators are especially recommended for heart and lung patients because they provide low resistance in exhaling air.
Donning and Doffing of N-95 Masks and Surgical Mask ${ }^{[8,9]}$

Donning (Putting on) an N95 mask

- Perform hand hygiene

- Hold the respirator in one hand, with the front of the respirator touching the inside of your hand. The metal nose piece should rest near the fingertips. Be sure the top and bottom straps of the respirator are hanging down below your hand and are not tangled or twisted

- Take the respirator and place it over your nose, mouth, and under the chin. Hold the respirator in place with one hand

- Use your free hand to place the straps on your head. It is very important the straps rest on the head and are not overlapping or tangled

- First, take the top strap of the respirator and place it just past the top of your head, above the ears

- Then take the bottom strap of the respirator and place it around the bottom of the head, just below the ears

- To ensure a proper seal of the respirator around the nose area, use the fingertips of both hands to bend the metal nose piece around the nose, starting in the center and working your way outward on each side.

Doffing (Taking off) an N95 mask

- Grasp the bottom strap of the mask and lift it over the head

- Then grasp the top strap of the mask and lift it over the head

- Dispose or reuse the mask per policy protocol

- Perform hand hygiene.

Donning Surgical mask

- Open the mask

- Prebend the nosepiece

- Place the mask on your face making sure to cover your nose

- Bring both top ties to the crown of your head and secure with a bow

- Tie bottom ties securely in a bow at the nape of your neck

- Once the mask is tied, press the malleable nose piece until a secure fit and good seal are achieved. This will reduce blow-by at the top of the mask

- Security check should be performed by checking the security of ties, nosepiece, and amount of air escaping from top, bottom, and sides of the mask.

\section{Doffing the surgical mask}

- Remove the mask by handling only the ties

- Untie the bottom, then the top tie

- Remove the mask from your face

- Properly dispose of the mask by touching only the ties. 
The illustrative diagrams for donning and doffing of surgical mask and N95 masks are shown in Figures 1 and 2.

\section{Recommendations for Extended Use/Reuse and Decontamination of N95 Respirators and Surgical Mask}

Ideally the N95 respiratory masks are only for single use, and this should be the standard practice whenever possible. CDC recommends extended use and reuse of N95 masks when there is a shortage of supplies during the pandemic. Extended use refers to wearing the same N95 respirator for repeated close contact encounters with several patients, without removing the respirator between patient encounters. Limited reuse refers to the practice of using the same N95 respirator for multiple encounters with patients but removing it after each encounter.

Extended use is favored over limited reuse because it is expected to involve less touching of the respirator and therefore less risk of contact transmission.

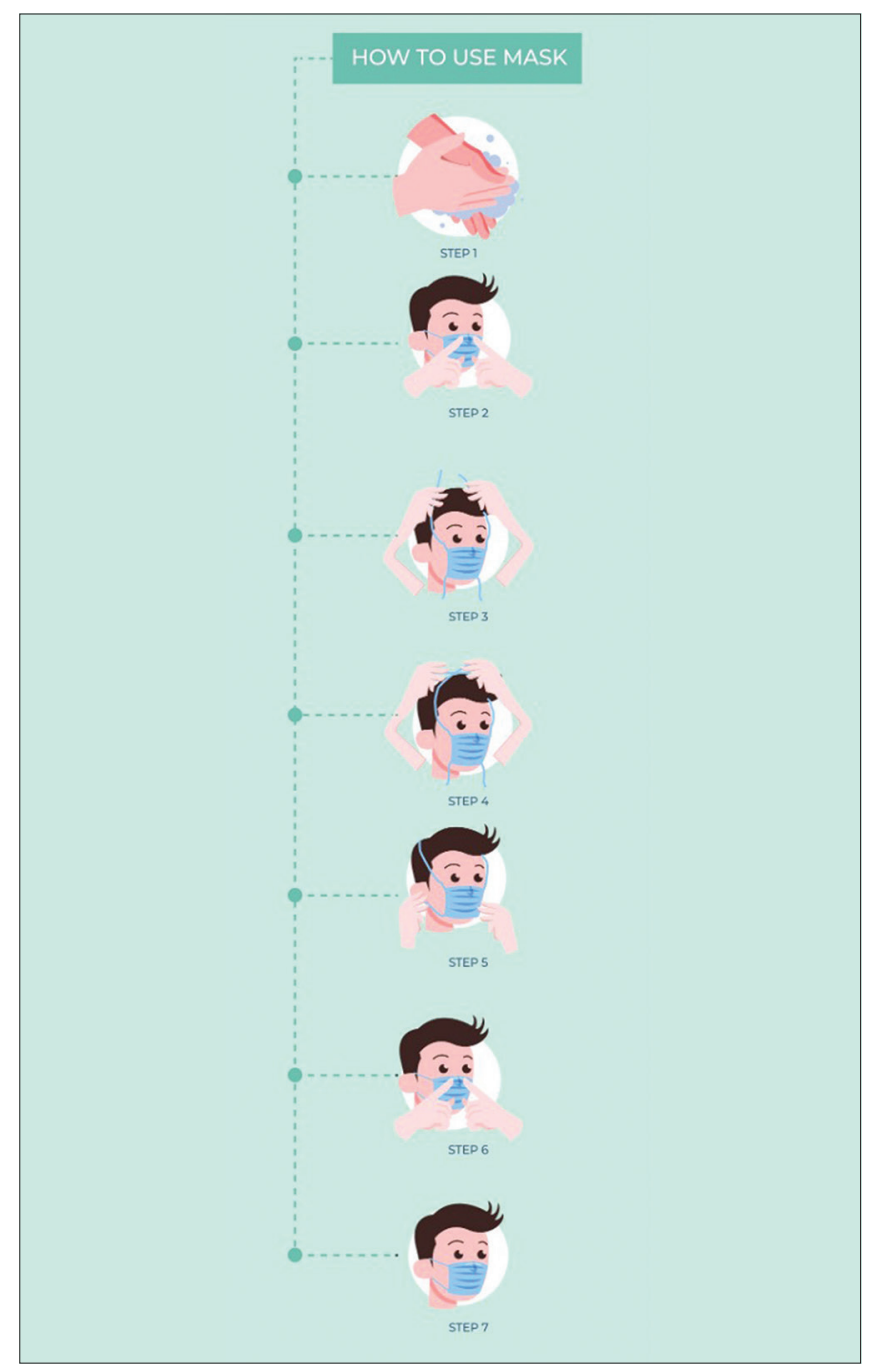

Figure 1: Donning surgical mask
Following points should be strictly implemented regarding reuse of N95 masks:

- Discard N95 respirators after aerosol-generating procedures

- Discard N95 respirators contaminated with blood and body fluids from patients

- Use a face shield or a surgical mask over an N95 respirator, when feasible to reduce surface contamination of the respirator

- Hang used respirators in a designated storage area or keep them in a clean, breathable container such as a paper bag between uses

- Avoid touching the inside of the respirator

- Limit the number of reuses to no more than five uses per respirator at a gap of at least $72 \mathrm{~h}$ before reusing again, i.e., N95 masks are to be reused after 3 days of placement in a paper bag [Figure 3]

- N95 respirators must only be used by a single wearer.

- Discard any respirator that is damaged or becomes hard to breathe through.

Risks associated with extended use and reuse of N95 respirators are risks of contact transmission, contamination of respirators with other pathogens and discomfort to wearer, and skin side effects like rash and ulcers.

Decontamination of N95 masks should be allowed only if there are severe crisis and shortage of supply. Decontamination might cause loss of filtration efficiency, poor fit and seal as a result of changes to the filtering material, straps, nose bridge material, or strap attachments. An ideal decontamination method should reduce the pathogen burden, maintain the integrity and function of the N95 respirator, and present no residual chemical hazard.

Most effective methods to decontaminate N95 masks are ultraviolet germicidal irradiation (UVGI), vaporous hydrogen peroxide (VHP), and moist heat. Reprocessing methods have not been validated by substantial research and there are currently no standardized methods or protocols for ensuring neither effectiveness nor integrity of the respirators after reprocessing. Numbers of reprocessing

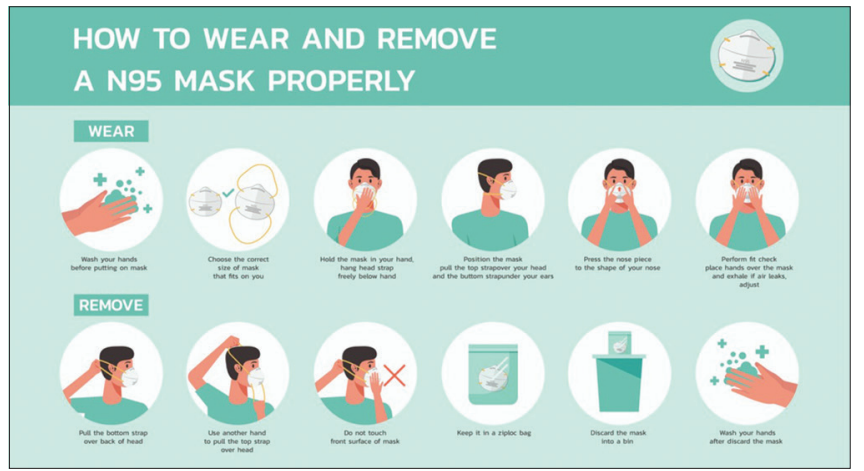

Figure 2: Donning and doffing N95 respirator 


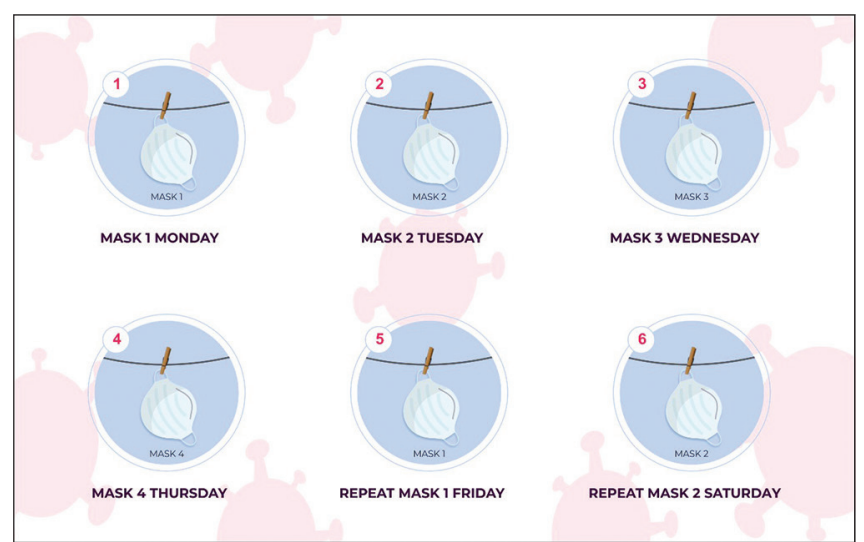

Figure 3: Reuse policy for N95 respirator

cycles are highly variable, depending on the reprocessing method used and the respirator brand/model.

(i) Ultraviolet germicidal irradiation (UVGI): The UV dose ranges from $60-950 \mathrm{~J} / \mathrm{cm}^{2}$ in the literature and can vary depending upon each Institutions standardization procedure. ${ }^{[10,11]}$ UVGI systems are relatively quick and easy to use, and do not leave chemical residues or risk exposing workers to toxic chemicals. However, ultraviolet light is harmful, so proper precautions are required to avoid UVGI exposure to skin or the eyes. There is a risk of degradation of the respirator material and reduced ability of the mask to filter out infectious bioaerosols after two to three cycles of decontamination. However, the effects of prolonged UVGI exposure after multiple decontamination cycles are still not known and it is unclear how much a cumulative dose of UVGI respirators can withstand. ${ }^{[12]}$

(ii) Vaporous hydrogen peroxide: STERRAD $100 \mathrm{~S}$ Gas Plasma Sterilizer $55 \mathrm{~min}$ standard cycle has been recommended as one of the methods of decontamination by WHO. ${ }^{[13]}$ Disadvantages of using vaporous hydrogen peroxide are cannot be used with any material containing celluloses and degradation in elastic straps after few cycles of decontamination. The most time-consuming part of this procedure is time required $(4 \mathrm{~h})$ before the decontamination room can be safely entered after fumigation. Both the UV light and hydrogen peroxide methods require equipment that not all hospitals have.

(iii) Moist heat: Moist heat, consisting of $60^{\circ} \mathrm{C}$ and $80 \%$ relative humidity have been tried by many researchers as this method causes minimal degradation in the filtration and fit performance. One limitation of the moist heat method is the uncertainty of the disinfection efficacy for various pathogens.

Extended use of surgical mask without removing up to $6 \mathrm{~h}$ is recommended. No quality evidence is available to date on surgical mask reprocessing and is not advised.
Comparison of FFP2, KN95, and N95 Filtering Facepiece Respirators ${ }^{[7]}$

The filter capacity (capacity to remove $\mathrm{x} \%$ of all particles that are $0.3 \mu \mathrm{m}$ in diameter or larger) of FFP2 respirator is $94 \%$, KN95 is $95 \%$ compared to N95 which is also at least 95\%. Hence, it is reasonable to consider China KN95, P2 (Australia/New Zealand AS/NZ P2), Korea $1^{\text {st }}$ Class KMOEL, and Japan DS2 FFRs as "similar" to US NIOSH N95 and European FFP2 respirators, for filtering nonoil-based particles. KN95 respirators are held in place by over-ear elastic loops, rather than behind the head elastics, resulting in a loose seal compared to N95 masks. Not all KN95 respirators meet the Chinese KN95 standards. However, with the current respirator shortage, unfortunately, the same applies for N95/FFP also.

\section{Cloth Face Mask}

CDC recommends wearing cloth face masks for general public at places where social distancing is difficult to maintain, especially in areas where there is a significant community-based transmission. Cloth face masks prevent the person wearing the mask from spreading respiratory droplets when talking, sneezing, or coughing especially in community settings like grocery stores, pharmacies, and gas stations. Cloth face mask or coverings can be made from household items. Children less than 2 years of age, and anyone who has difficulty in breathing, unconscious, unable to remove the mask without assistance should not wear cloth mask. ${ }^{[14]}$ The cloth face masks are not comparable to surgical masks or N95 respirators in terms of filtering capacity, however are economical, can be made anywhere by anyone, reusable, and easily available. Maximum protection from catching the infection from others by the aerosol route offered by cloth mask is around $50 \%-70 \%$, compared to the N95 mask (99\%) and surgical mask which offers $75 \%-80 \%$ protection.

Recently, Indian Institute of Technology (IIT) Delhi and its start-up "ETEX" has developed an affordable alternative mask, "Kawach." The "Kawach" mask, developed by ETEX, is at par with N95 in terms of fitting and filtration efficiency. It provides up to $98 \%$ filtration efficiency up to $3-\mu \mathrm{m}$ size particle and is economical being priced at Rs $45 .{ }^{[15]}$

\section{Face Shields}

A face shield is classified as PPE that provides barrier protection to the facial area and mucous membranes of eyes, nose, and lips. An ideal face shield should cover the forehead, extend below the chin, and wrap around the side of the face [Figure 4]. The advantages of using face shield are many: more comfortable, protect a larger portion of the face, less fogging than goggles, less claustrophobic, no impact on breathing resistance, no fit testing required, can 


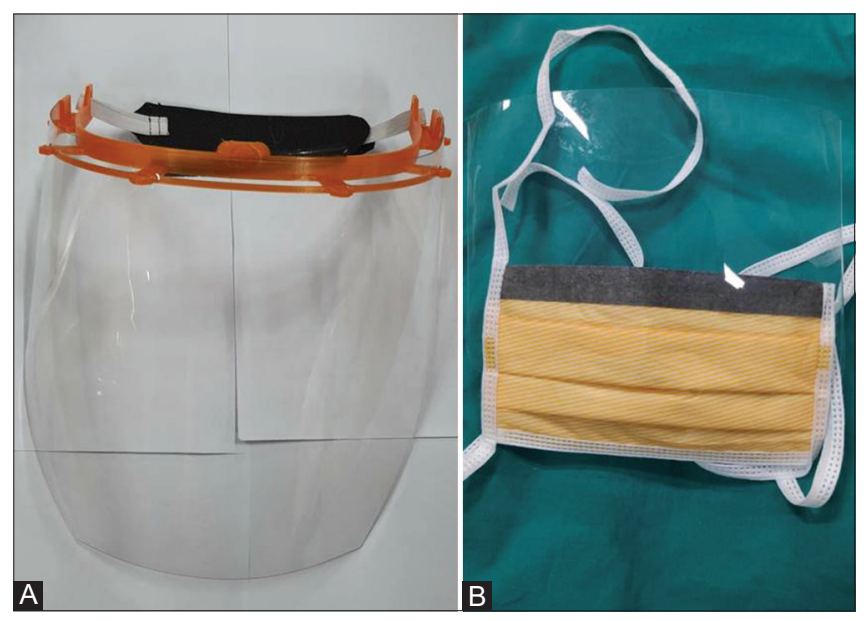

Figure 4 (A and B): (A) Face shield and (B) mask with integral visor

be disinfected easily, wearers do not need to be clean shaven, easy to don and doff, relatively inexpensive, no impact on vocalization, can be worn concurrent to other face/eye PPE, do not impede facial nonverbal communication, protects against self-inoculation over a wider facial area, and may extend the useful life of a protective facemask when used concurrently. Disadvantages of using a face shield are glare effects, fogging, may be optically imperfect, some models may not fit properly over some respirators, and may be bulkier than goggles. The major structural components of face shield are (i) visor, (ii) frame, and (iii) suspension straps. Since face shield is not a tight facial fit, it cannot be used as a replacement for a mask. Face shields provide a barrier to body fluids and are commonly used as an alternative to goggles as they confer protection to a larger area of the face.

Various studies have reported $96 \%$ and $92 \%$ reductions in the risk of inhalational exposure immediately after a cough for a face shield at distances of 18 in $(46 \mathrm{~cm})$ and 72 in (183 $\mathrm{cm})$, respectively. ${ }^{[16,17]}$ The World Health Organization recommends the use of a face shield as an alternative to the use of a surgical mask with goggles ${ }^{[18]}$ In Radiology Department, face shield can be used alone when a splash of body fluid is expected like during drainage procedures. During COVID crisis, surgical mask with face shield or mask with integral visor is preferable in imaging COVID suspect cases maintaining adequate social distancing and hand hygiene practices. However, when imaging COVID positive patients, face shield is an integral part of full PPE and should be worn along with an N95 respirator. In emergency situations, at times of crisis, WHO recommends the use of isolated face shield with proper design in the absence of surgical masks.

\section{Conclusion}

To conclude, this article highlights the importance and appropriate usage of cloth mask, N95 masks, surgical masks, and face shields in routine radiology practice which is extremely essential during COVID-19 crisis. Face shields cannot be used as a replacement for a mask. Cloth face mask should not be used by the healthcare worker in the hospital setting and is not to be considered a substitute for N95 mask or surgical mask. N95 masks should be reserved for COVID suspect and positive cases. Surgical mask should only be used for routine cases in which there is no suspicion for COVID infection. Only in crisis times and nonavailability of N95 masks, surgical mask with visor may be considered for examining COVID suspect cases with all due precautions.

Binit Sureka, Sanjeev Misra

Associate Professor, Deputy Medical Superintendent, Departments of Diagnostic and Interventional Radiology, 'Director and CEO, All India Institute of Medical Sciences, Basni, Jodhpur, Rajasthan, India. E-mail: binitsurekapgi@gmail.com

\section{References}

1. Lee SA, Grinshpun SA, Reponen T. Respiratory performance offered by N95 respirators and surgical masks: Human subject evaluation with $\mathrm{NaCl}$ aerosol representing bacterial and viral particle size range. Ann Occup Hyg 2008;52:177-85.

2. How surgical masks are made. [Internet]. Available from: Available from: https://www.thomasnet.com/articles/other/howsurgical-masks-are-made/. [Last cited on 2020 Apr 18].

3. $3 \mathrm{M}$ Masks \& Respirators for Personal Health Care. [Internet]. Available from: https://multimedia. $3 \mathrm{~m} . c 0 \mathrm{~m} / \mathrm{mws} /$ media/1425070O/3m-particulate-respirator-8210-n95-technical-s pecifications.pdf. [Last cited on 2020 Apr 18].

4. National Institute for Occupational Safety and Hygiene (NIOSH) NIOSH Guide to the Selection and Use of Particulate Respirators Certified Under 42 CFR 84. Cincinnati, Ohio, USA: National Institute for Occupational Safety and Hygiene (NIOSH); 1996. (DHHS (NIOSH) Publication no. 96-101).

5. Centres for Disease Control and Prevention. [Internet]. Available from: https://www.cdc.gov/niosh/npptl/pdfs/Underst andDifferenceInfographic-508.pdf. [Last cited on 2020 Apr 18].

6. Radiological Society of North America [Internet]. Available from: https://www.rsna.org/-/media/Files/RSNA/Covid-19/RSNACOVID-19-bestpractices.ashx?la=en\&hash=58700DDDEDB3E5A 9C8EDE80BE534B4ABB10291B7. [Last cited on 2020 May 09].

7. Fast Life Hacks. [Internet]. Available from: https://fastlifehacks. com/n95-vs-ffp/\#N95_vs_FFP3_FFP2. [Last cited on 2020 May 27].

8. Centres for Disease Control and Prevention. [Internet]. Available from: https://www.cdc.gov/vhf/ebola/hcp/ppe-training/ n95respirator_gown/donning_09.html. [Last cited on 2014 Oct 29].

9. Registered Nurse [Internet] [cited]. Available from: https:// www.registerednursern.com/how-to-don-and-doff-n-95-maskrespirator/. [Last cited on 2020 May 10].

10. Fisher EM, Shaffer RE. A method to determine the available UV-C dose for the decontamination of filtering facepiece respirators. J Appl Microbiol 2011;110:287-95.

11. Centres for Disease Control and Prevention. [Internet]. Available from: https://www.cdc.gov/coronavirus/2019-ncov/hcp/ ppe-strategy/decontamination-reuse-respirators.html. [Last cited on 2020 Apr 03].

12. Lindsley WG, Martin SB Jr, Thewlis RE, Sarkisian K, Nwoko JO, Mead KR, et al. Effects of ultraviolet germicidal irradiation (UVGI) on N95 respirator filtration performance and structural integrity. J Occup Environ Hyg 2015;12:509-17. 
13. World Health Organization. [Internet]. Available from: https:// www.who.int/publications-detail/rational-use-of-personal-prote ctive-equipment-for-coronavirus-disease-(covid-19)-and-conside rations-during-severe-shortages. [Last cited on 2020 Apr 06].

14. Centres for Disease Control and Prevention. [Internet]. Available from: https://www.cdc.gov/coronavirus/2019-ncov/preventgetting-sick/cloth-face-cover.html. [Last cited on 2020 Apr 03].

15. The Logical Indian [Internet]. Available from:https://thelogicalindian. com/news/iit-delhi-launches-masks-for-rs-45-like-n95-20702. [Last cited on 2020 Apr 21].

16. Lindsley WG, Noti JD, Blachere FM, Szalajda JV, Beezhold DH. Efficacy of face shields against cough aerosol droplets from a cough simulator. J Occup Environ Hyg 2014;11:509-18.

17. Roberge RJ. Face shields for infection control: A review. J Occup Environ Hyg 2016;13:235-42.

18. World Health Organization: Aide Memoire. Standard Precautions in Healthcare. [Internet]. Available from: http://www.who.int/ csr/resources/publications/EPR_AM2_E7.pdf. [Last cited on 2015 Jul 15].
This is an open access journal, and articles are distributed under the terms of the Creative Commons Attribution-NonCommercial-ShareAlike 4.0 License, which allows others to remix, tweak, and build upon the work non-commercially, as long as appropriate credit is given and the new creations are licensed under the identical terms.

\begin{tabular}{|l|l|}
\hline \multicolumn{2}{|c|}{ Access this article online } \\
\hline Quick Response Code: & \\
\hline & Website: \\
\hline & www.ijri.org \\
\cline { 2 - 3 } & DOl: \\
& 10.4103/ijri.IJRI_350_20 \\
\hline
\end{tabular}

Cite this article as: Sureka B, Misra S. N95 respirator or triple layer surgical mask: Radiologist perspective. Indian J Radiol Imaging 2021;31:S198-203.

Received: 09-May-2020

Revised: 29-May-2020

Accepted: 05-Jul-2020
Published: 23-Jan-2021 